\title{
The Promising Role of Lung Ultrasound in Assessment of Volume Status for Patients Receiving Maintenance Renal Replacement Therapy
}

\author{
Abhilash Koratala ${ }^{a, b} \quad$ Claudio Ronco ${ }^{c, d}$ Amir Kazory ${ }^{a}$ \\ ${ }^{a}$ Division of Nephrology, Hypertension and Renal Transplantation, University of Florida, Gainesville, FL, USA; ${ }^{b}$ Division of \\ Nephrology, Medical College of Wisconsin, Milwaukee, WI, USA; ${ }^{C}$ Department of Nephrology, San Bortolo Hospital and \\ International Renal Research Institute of Vicenza (IRRIV), Vicenza, Italy; ${ }^{\mathrm{d}}$ Department of Medicine, University of Padova, \\ Padova, Italy
}

Patients with end-stage renal disease (ESRD) receiving maintenance dialysis therapy portend a unique cardiovascular risk profile. Sustained volume overload has been identified as a potent and independent risk factor for all-cause and cardiovascular mortality in this patient population $[1,2]$. Due to limitations of physical exam in precise assessment of volume status, a multitude of objective methods (e.g., natriuretic peptides, blood volume monitoring, and bioimpedance spectroscopy) have been explored to guide the ultrafiltration process during dialysis therapy. Unfortunately, these methods have serious limitations and so far, none of them has shown promising results when used in isolation. Point-of-care ultrasonography has recently emerged as an intriguing adjunct to physical examination [3]. Lung ultrasound (LUS), a noninvasive, radiation-free technique to estimate extravascular lung water (EVLW), has been increasingly recognized as an invaluable addition to the armamentarium of the nephrologists. Herein, we provide a brief overview of the role of LUS in the assessment of volume stats in patients receiving dialysis.

$\begin{aligned} & \text { karger@karger.com } \\ & \text { www.karger.com/bpu }\end{aligned}$
Karger $\%$

\section{What Is the Diagnostic Utility of LUS for Detection of Pulmonary Congestion?}

The key advantage of performing bedside LUS is its ability to diagnose pulmonary congestion before it is clinically evident [4]. It has been long recognized that conventional physical examination poorly reveals interstitial lung edema in ESRD. For instance, in a study including 79 patients treated with hemodialysis, the sensitivity of lung crackles, peripheral edema, and their combination were only 9,3 , and $13 \%$, respectively, to detect congestion [5]. In another study, $57 \%$ of the patients treated with peritoneal dialysis who had severe lung congestion $(>30$ B-lines) presented with no dyspnea [6].

\section{Is Asymptomatic Lung Congestion Important?}

Both symptomatic and asymptomatic lung congestion have been associated with worse outcomes in patients with ESRD. In a multicenter study including 392 patients receiving hemodialysis, those with very severe congestion 
on 28-zone LUS had a 4-fold risk of death and a 3-fold risk of cardiac events. The relative risk of mortality was increased by $70 \%$ in patients with moderate-to-severe congestion as well. Of particular note, $71 \%$ of the patients in this group were asymptomatic or presented mild symptoms of heart failure [7]. Similarly, a prospective study reported that patients with presence of B-lines in $\geq 21.4 \%$ of the scan zones after 2 consecutive hemodialysis sessions were more likely to be hospitalized for cardiac causes [8].

Increased EVLW has also been shown to negatively affect the physical functioning of patients receiving hemodialysis or peritoneal dialysis $[9,10]$.

\section{What Is the Benefit of LUS-Guided Dry Weight Adjustment?}

As the number of B-lines dynamically changes with ultrafiltration, the residual congestion at the end of dialysis treatment can be used to titrate dry weight $[11,12]$. In a recent randomized controlled trial including 71 clinically euvolemic patients, Loutradis et al. [13] found that patients who underwent dry weight reduction guided by pre-hemodialysis LUS had greater weight reduction compared to those receiving standard care, correlating with the change in number of B-lines $(-5.3$ in active group vs. +2.2 in controls). Moreover, at 8 weeks, the magnitude of 48-h ambulatory blood pressure reduction was significantly greater in the active group (systolic -6.61 vs. $-0.67 \mathrm{~mm} \mathrm{Hg}$ and diastolic -3.85 vs. $-0.55, p=$ 0.033 ). Furthermore, the active group demonstrated a significant reduction in left ventricular filling pressures at 8 weeks [14].

\section{What Are the Technical Considerations of Bedside Lung Ultrasonography?}

Evaluation of an air-rich organ such as lung is an inherent limitation of ultrasound technique because air scatters the ultrasound beam and does not allow proper visualization of the underlying structures. However, this limitation actually turns into a diagnostic advantage when performing LUS because of the characteristic artifacts (i.e., A- and B-lines). In normal aerated lung, Alines are seen as hyperechoic, horizontal lines arising at regular intervals from the pleural line. These are reverberation artifacts formed due to multiple reflections of the ultrasound beam between the transducer and the pleura with air-filed lung underneath. B-lines are seen in the setting of interstitial edema or thickened interlobular septae, which are defined as discrete laser-like vertical hyperechoic artifacts that arise from the pleural line, extend to the end of the image without fading, and move synchronously with lung sliding [15]. The total number of B-lines provides an estimate of the amount of EVLW and aids in the determination of volume status as well as monitoring response to therapy [16]. Most studies in ESRD utilized a 28-zone scanning technique to obtain the total B-line count, which involves scanning of the anterior and lateral chest from the second to fourth (on the right side to the fifth) intercostal spaces and from the parasternal to midaxillary lines bilaterally [17] (Fig. 1). Description of other sonographic lung pathologies such as pleural effusion and consolidation is beyond the scope of this article.

LUS is a relatively easy-to-learn technique for physicians with basic understanding of the ultrasound physics. In fact, Gargani et al. [18] have demonstrated that nephrologists can be effectively trained to measure sonographic lung congestion by an entirely web-based program. However, like with any other skill, continued practice is the key to achieving mastery. Integration of point-of-care ultrasonography into nephrology fellowship curricula [19] with longitudinal training aids in long-term skill retention.

\section{Future Directions}

Current evidence clearly suggests that LUS is an effective bedside tool to optimize the volume status in patients with ESRD. Nevertheless, the question whether targeting a certain B-line score on LUS improves the overall prognosis of these patients remains unanswered. A multicenter clinical trial currently in progress (Lung Water by Ultrasound Guided Treatment to Prevent Death and Cardiovascular Complications in High-Risk ESRD Patients with Cardiomyopathy [LUST]) is expected to provide some valuable insights [20]. Future studies should also investigate the utility of simplified LUS protocols such as the 4-zone scanning technique [21] as an alternative to the traditional 28zone method to possibly facilitate its more widespread acquisition.

\section{Acknowledgments}

None. 

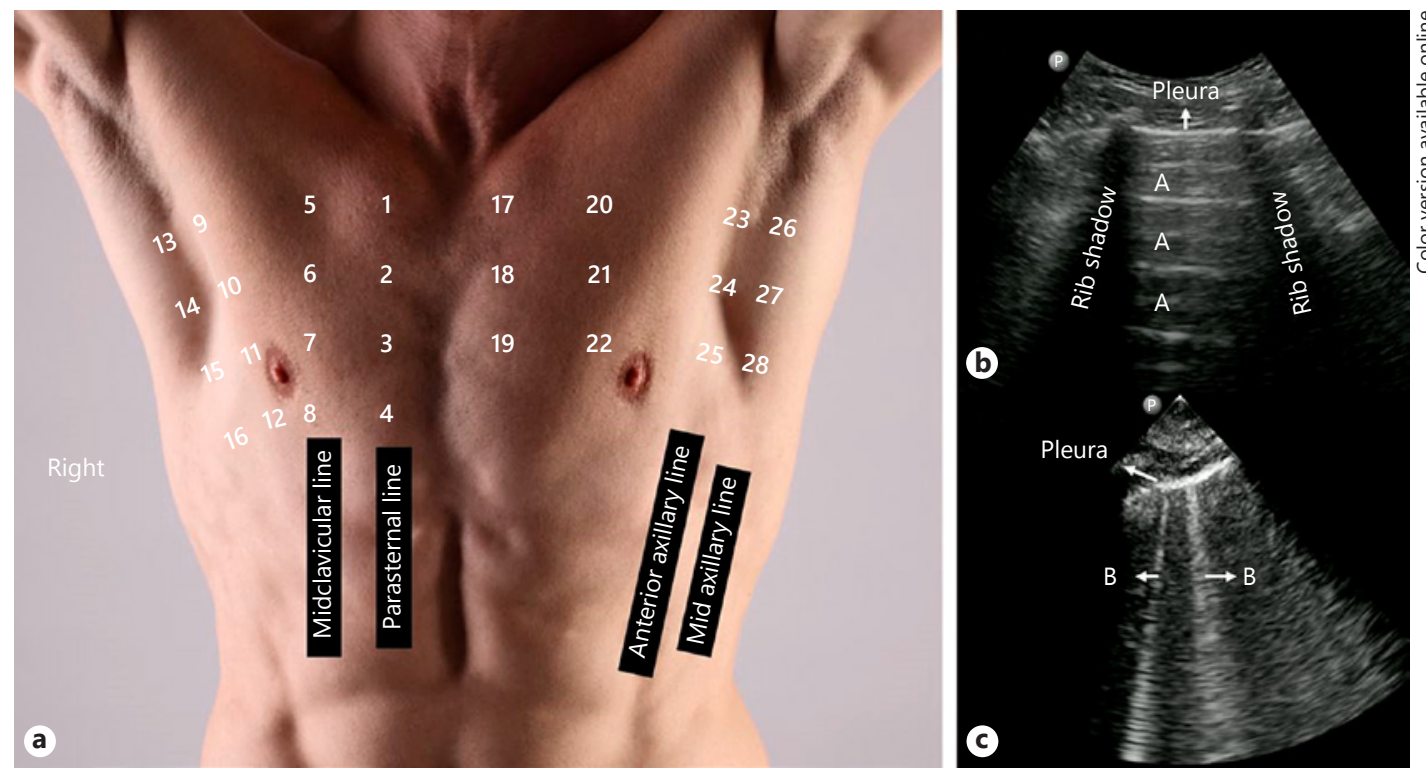

Fig. 1. a Illustration of the 28 scan zones used for lung ultrasonography. The technique involves scanning of the anterior and lateral chest from the second to fourth (on the right side to the fifth) in- tercostal spaces and from the parasternal to midaxillary lines bilaterally; (b) A-lines on normal LUS; (c) B-lines on LUS suggestive of increased EVLW.

\section{Statement of Ethics}

Human and animal rights: this article does not contain any studies with human participants or animals performed by any of the authors.

\section{Disclosure Statement}

The authors have declared that no conflict of interest exists.

\section{Funding Sources}

There is no funding to declare.

\section{Author Contributions}

A. Koratala designed and drafted the manuscript. C.R. and A. Kazory revised the manuscript critically for important intellectual content. All authors have read and approved the manuscript prior to final submission.

\section{References}

1 Zoccali C, Moissl U, Chazot C, Mallamaci F, Tripepi G, Arkossy O, et al. Chronic Fluid Overload and Mortality in ESRD. J Am Soc Nephrol. 2017 Aug;28(8):2491-7.

2 Agarwal R. Volume overload in dialysis: the elephant in the room, no one can see. Am J Nephrol. 2013;38(1):75-7.

3 Narula J, Chandrashekhar Y, Braunwald E. Time to Add a Fifth Pillar to Bedside Physical Examination: Inspection, Palpation, Percussion, Auscultation, and Insonation. JAMA Cardiol. 2018 Apr;3(4):346-50.

4 Picano E, Scali MC, Ciampi Q, Lichtenstein D. Lung Ultrasound for the Cardiologist. JACC Cardiovasc Imaging. 2018 Nov;11(11): 1692-705

5 Torino C, Gargani L, Sicari R, Letachowicz K, Ekart R, Fliser D, et al. The Agreement between Auscultation and Lung Ultrasound in
Hemodialysis Patients: the LUST Study. Clin J Am Soc Nephrol. 2016 Nov;11(11):200511.

6 Panuccio V, Enia G, Tripepi R, Torino C, Garozzo $\mathrm{M}$, Battaglia GG, et al. Chest ultrasound and hidden lung congestion in peritoneal dialysis patients. Nephrol Dial Transplant. 2012 Sep;27(9):3601-5.

7 Zoccali C, Torino C, Tripepi R, Tripepi G, D'Arrigo G, Postorino M, et al.; Lung US in CKD Working Group. Pulmonary congestion predicts cardiac events and mortality in ESRD. J Am Soc Nephrol. 2013 Mar;24(4): 639-46.

8 Beaubien-Souligny W, Rhéaume M, Blondin MC, El-Barnachawy S, Fortier A, Éthier J, et al. A Simplified Approach to Extravascular Lung Water Assessment Using Point-of-Care Ultrasound in Patients with End-Stage Chronic
Renal Failure Undergoing Hemodialysis. Blood Purif. 2018;45(1-3):79-87.

9 Enia G, Tripepi R, Panuccio V, Torino C, Garozzo M, Battaglia GG, et al. Pulmonary congestion and physical functioning in peritoneal dialysis patients. Perit Dial Int. 2012 SepOct:32(5):531-6.

10 Enia G, Torino C, Panuccio V, Tripepi R, Postorino M, Aliotta R, et al.; Lung Comets Cohort Working Group. Asymptomatic pulmonary congestion and physical functioning in hemodialysis patients. Clin J Am Soc Nephrol. 2013 Aug;8(8):1343-8.

11 Noble VE, Murray AF, Capp R, Sylvia-Reardon MH, Steele DJ, Liteplo A. Ultrasound assessment for extravascular lung water in patients undergoing hemodialysis. Time course for resolution. Chest. 2009 Jun;135(6):14339. 
12 Koratala A, Chamarthi G, Kazory A. Pointof-Care Ultrasonography for Objective Volume Management in End-Stage Renal Disease. Blood Purif. 2019 Oct;9:1-5.

13 Loutradis C, Sarafidis PA, Ekart R, Papadopoulos C, Sachpekidis V, Alexandrou ME, et al. The effect of dry-weight reduction guided by lung ultrasound on ambulatory blood pressure in hemodialysis patients: a randomized controlled trial. Kidney Int. 2019 Jun; 95(6):1505-13.

14 Loutradis C, Papadopoulos CE, Sachpekidis V, Ekart R, Krunic B, Karpetas A, et al. Lung Ultrasound-Guided Dry Weight Assessment and Echocardiographic Measures in Hypertensive Hemodialysis Patients: A Randomized Controlled Study. Am J Kidney Dis. 2020 Jan;75(1):11-20.

15 Volpicelli G, Elbarbary M, Blaivas M, Lichtenstein DA, Mathis G, Kirkpatrick AW, et al.; In- ternational Liaison Committee on Lung Ultrasound (ILC-LUS) for International Consensus Conference on Lung Ultrasound (ICC-LUS). International evidence-based recommendations for point-of-care lung ultrasound. Intensive Care Med. 2012 Apr;38(4):577-91.

16 Agricola E, Bove T, Oppizzi M, Marino G, Zangrillo A, Margonato A, et al. "Ultrasound comet-tail images": a marker of pulmonary edema: a comparative study with wedge pressure and extravascular lung water. Chest. 2005 May;127(5):1690-5.

17 Frassi F, Gargani L, Tesorio P, Raciti M, Mottola G, Picano E. Prognostic value of extravascular lung water assessed with ultrasound lung comets by chest sonography in patients with dyspnea and/or chest pain. J Card Fail. 2007 Dec;13(10):830-5.

18 Gargani L, Sicari R, Raciti M, Serasini L, Passera M, Torino C, et al. Efficacy of a re- mote web-based lung ultrasound training for nephrologists and cardiologists: a LUST trial sub-project. Nephrol Dial Transplant. 2016 Dec;31(12):1982-8.

19 Koratala A, Segal MS, Kazory A. Integrating Point-of-Care Ultrasonography Into $\mathrm{Ne}$ phrology Fellowship Training: A Model Curriculum. Am J Kidney Dis. 2019 Jul;74(1):15.

20 Lung Water by Ultrasound Guided Treatment in Hemodialysis Patients (The Lust Study). Available from: https://clinicaltrials. gov/ct2/show/NCT02310061. Last accessed: $12 / 1 / 2019$.

21 Platz E, Campbell RT, Claggett B, Lewis EF, Groarke JD, Docherty KF, et al. Lung Ultrasound in Acute Heart Failure: Prevalence of Pulmonary Congestion and Short- and LongTerm Outcomes. JACC Heart Fail. 2019 Oct; 7(10):849-58. 